\title{
Weekly Teriparatide for Delayed Unions of Atypical Subtrochanteric Femur Fractures
}

\author{
Fumio Fukuda $\cdot$ Naoaki Kurinomaru $\cdot$ Akihiko Hijioka
}

To view enhanced content go to www.biologicstherapy-open.com

Received: November 30, 2013 / Published online: January 29, 2014

(c) The Author(s) 2014. This article is published with open access at Springerlink.com

\section{ABSTRACT}

Introduction: The occurrence of atypical femur fractures (AFFs) in patients on prolonged bisphosphonate treatment has been gaining medical attention, but the use of pharmacotherapy for these fractures has not been explored in detail. The authors describe a case of AFFs successfully treated with once-weekly administration of $56.5 \mu \mathrm{g}$ teriparatide (TPTD).

Case Presentation: The patient was a 74-yearold female patient who had been taking alendronate for approximately 6 years and who suffered with a fall while walking. X-rays revealed a subtrochanteric right femur fracture. The contralateral femur showed cortical thickening and a transverse radiolucent fracture line. Based on these specific features, the patient was diagnosed with AFF. The patient underwent osteosynthesis with intramedullary

Electronic supplementary material The online version of this article (doi:10.1007/s13554-014-0013-5) contains supplementary material, which is available to authorized users.

F. Fukuda $(\bowtie) \cdot$ N. Kurinomaru · A. Hijioka

Kitakyushu General Hospital,

5-10-10 Kokura-Minami,

Kitakyushu 800-0295, Japan

e-mail: f-fukuda@kitakyu-hp.or.jp nailing for the right fracture. Alendronate treatment was discontinued. Low-intensity pulsed ultrasonography therapy did not affect the healing of the fracture with delayed union, even after 3 months of application. Prophylactic osteosynthesis was performed for the subtrochanteric left femur. Bone tissue collected from the left fracture site during surgery showed severe suppression of bone turnover. Union of bilateral femurs was achieved after 3 months of a once-weekly administration of TPTD.

Conclusion: Once-weekly TPTD treatment is shown to be beneficial for improving the healing of AFFs showing delayed union.

Keywords: Atypical femoral fractures; Bisphosphonates; Delayed union; Teriparatide

\section{INTRODUCTION}

Atypical subtrochanteric or femoral shaft fractures occasionally show delayed union, especially in osteoporosis patients undergoing long-term treatment with bisphosphonates. However, there have been few published studies on the effects of pharmacotherapy on 
these fractures. Previous studies have suggested that teriparatide (TPTD) therapy can be effective to promote healing of fractures with delayed unions [1-6]. However, it is still unknown whether a once-weekly administration of TPTD could offer an effective alternative to the daily TPTD. Herein, the authors report a patient with delayed union after surgery for an atypical femoral fracture (AFF) who was effectively treated with once-weekly administration of $56.5 \mu \mathrm{g}$ of TPTD.

\section{CASE REPORT}

A 74-year-old female patient with osteoporosis, who had been taking alendronate and alfacalcidol for approximately 6 years, suffered femur fractures following a fall in March 2012. Radiography revealed a subtrochanteric right femur fracture (Fig. 1a). In addition, her contralateral thigh showed thickening of the lateral cortex and a transverse radiolucent fracture line in the subtrochanter (Fig. 1b). The patient was diagnosed with AFFs. The patient had normal levels of bone resorption markers, but low levels of a bone formation marker (Table 1).

Four days after admission, the authors performed minimally invasive osteosynthesis using intramedullary nails (Fig. 2). Alfacalcidol was continued but alendronate was ceased. Two weeks later, low-intensity pulsed ultrasonography (LIPUS) therapy was started for both thigh regions to promote bone healing. In June 2012, radiography and computed tomography imaging revealed signs of delayed union at the right fracture site (Fig. 3).

The patient could not walk without a crutch owing to contralateral thigh pain. After obtaining informed consent, prophylactic osteosynthesis was performed on the left subtrochanteric lesion in June 2012. Bone biopsy samples collected at the intramedullary nail insertion site revealed an absence of osteoblasts and osteoclasts on trabecular surfaces (Fig. 4), as well as low bone volume (Table 2).

LIPUS treatment was restarted after surgery. In August 2012, once-weekly TPTD (56.5 $\mu \mathrm{g})$
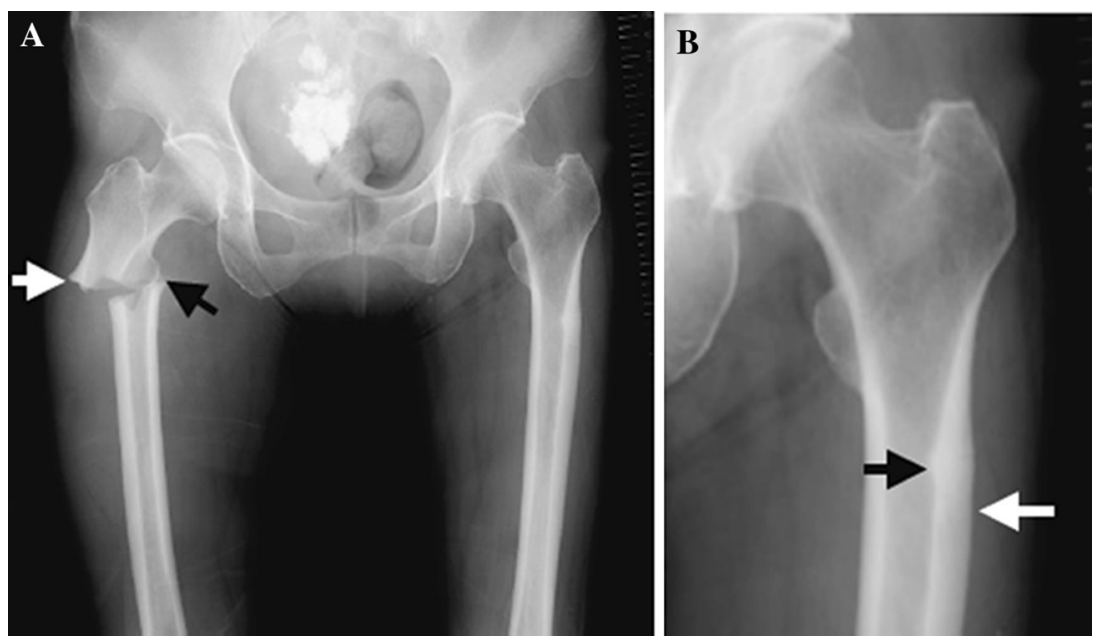

Fig. 1 Radiograph imaging at the time of admission. a Radiograph imaging at the time of admission shows the right subtrochanteric fracture with breaking of the lateral cortex (white arrow) and spiking of the medial cortex (black arrow). $\mathbf{b}$ Zoomed image of the left thigh shows thickening of the lateral cortex (white arrow) and a transverse radiolucent fracture line (black arrow) 
Table 1 Biochemical markers at the time of admission

\begin{tabular}{lll}
\hline Parameters & Results & $\begin{array}{l}\text { Reference } \\
\text { range }\end{array}$ \\
\hline Serum calcium $(\mathrm{mg} / \mathrm{dL})$ & 8.9 & $8.7-10.3$ \\
Serum phosphorous $(\mathrm{mg} / \mathrm{dL})$ & 2.7 & $2.5-4.7$ \\
Serum alkaline phosphatase $(\mathrm{U} / \mathrm{L})$ & 163 & $115-369$ \\
Urine NTX (nmol BCE/ & 17.1 & $14.3-89.0$ \\
$\quad$ mmol·Cre) & & \\
Serum TRACP-5b $(\mathrm{mU} / \mathrm{dL})$ & 257 & $120-420$ \\
Serum P1NP $(\mu \mathrm{g} / \mathrm{L})$ & 6.4 & $17.0-64.7^{\mathrm{a}}$ \\
Serum ucOC $(\mathrm{ng} / \mathrm{mL})$ & 0.39 & $<4.50$ \\
Serum homocysteine $(\mathrm{nmol} / \mathrm{mL})$ & 8.2 & $5.1-11.7$ \\
Serum pentosidine $(\mathrm{pg} / \mathrm{mL})$ & 20.3 & $9.2-43.1$ \\
\hline
\end{tabular}

$N T X N$-telopeptide of type I collagen, $P 1 N P$ procollagen type $1 N$-terminal propeptide, TRACP-5b tartrate-resistant acid phosphatase type $5 \mathrm{~b}, u c O C$ uncarboxylated osteocalcin a Postmenopausal

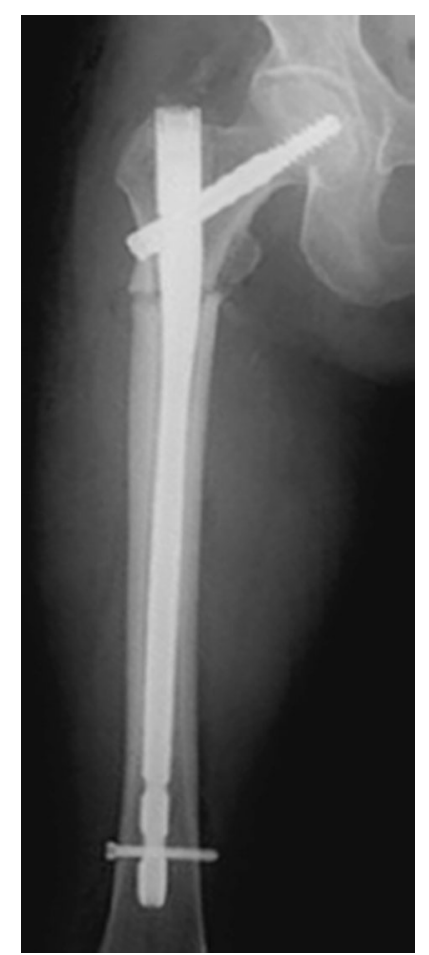

Fig. 2 Radiograph imaging of the right thigh at the time of surgery
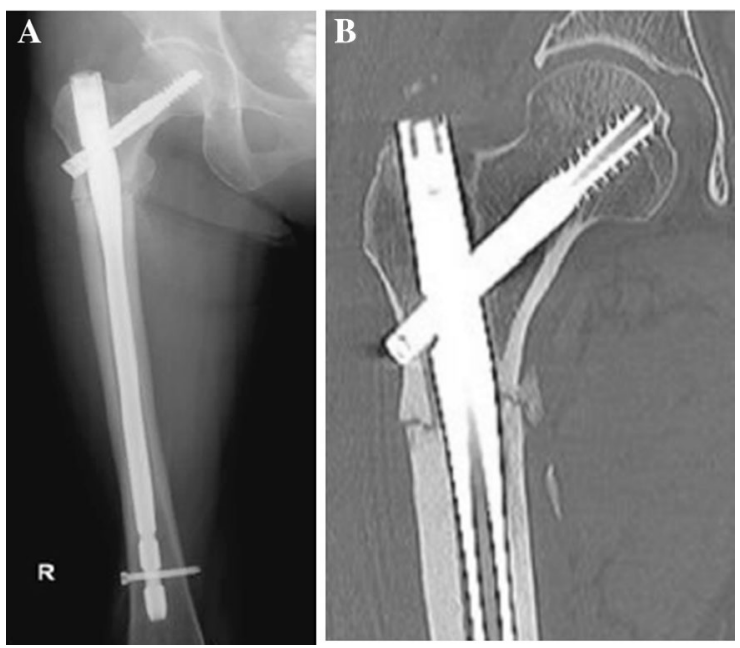

Fig. 3 Radiograph and CT imaging after 3 months of LIPUS therapy. a Radiograph imaging after 3 months of LIPUS therapy shows delayed union at the fracture site. b CT imaging after 3 months of LIPUS therapy shows delayed union at the fracture site. $C T$ computerized tomography, LIPUS low-intensity pulsed ultrasonography

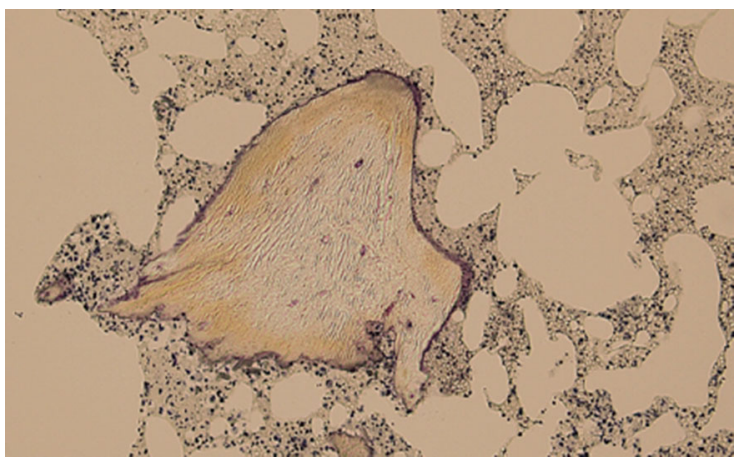

Fig. 4 Trabecular surfaces ( $\times 200$ magnification, Villanueva bone stain) show a total absence of osteoblasts and osteoclasts (photomicrographs were taken at the Niigata Bone Science Institute, Niigata, Japan)

was added, and the patient's tartrate-resistant acid phosphatase type 5b (TRACP-5b) and procollagen type $1 \mathrm{~N}$-terminal propeptide (P1NP) levels increased transiently, as did their lumbar bone mineral density (Table 3). After 3 months of TPTD treatment, the patient's fractures had improved (Fig. 5). Administration of TPTD was terminated in February 2013 and 
Table 2 Results of histomorphometric assessment of bone biopsy specimens

\begin{tabular}{|c|c|c|c|}
\hline Parameters & Abbreviations & Results & $\begin{array}{l}\text { Reference } \\
\text { data }^{\mathbf{a}}\end{array}$ \\
\hline \multicolumn{4}{|l|}{ Bone volume } \\
\hline Bone volume & $\mathrm{BV} / \mathrm{TV}(\%)$ & 11.54 & $11.0 \pm 1.8$ \\
\hline $\begin{array}{l}\text { Trabecular } \\
\text { thickness }\end{array}$ & $\operatorname{Tb} . T h(\mu \mathrm{m})$ & 103.09 & $131.3 \pm 28.1$ \\
\hline Wall thickness & W.Th $(\mu \mathrm{m})$ & 17.96 & $28.3 \pm 3.7$ \\
\hline \multicolumn{4}{|c|}{ Osteoid characteristics } \\
\hline $\begin{array}{l}\text { Osteoid } \\
\text { volume }\end{array}$ & OV/TV (\%) & 0.01 & $0.36 \pm 0.31$ \\
\hline $\begin{array}{l}\text { Osteoid } \\
\text { volume }\end{array}$ & $\mathrm{OV} / \mathrm{BV}(\%)$ & 0.06 & $1.6 \pm 0.4$ \\
\hline $\begin{array}{l}\text { Osteoid } \\
\text { surface }\end{array}$ & OS/BS (\%) & 1.04 & $12.8 \pm 2.2$ \\
\hline $\begin{array}{l}\text { Osteoid } \\
\text { thickness }\end{array}$ & O.Th $(\mu \mathrm{m})$ & 2.83 & $6.4 \pm 0.8$ \\
\hline $\begin{array}{l}\text { Osteoblast } \\
\text { surface }\end{array}$ & Ob.S/BS (\%) & NC & \\
\hline \multicolumn{4}{|l|}{ Resorption } \\
\hline Eroded surface & ES/BS (\%) & 0.06 & $3.0 \pm 1.0$ \\
\hline $\begin{array}{l}\text { Osteoclast } \\
\text { surface }\end{array}$ & Oc.S/BS (\%) & $\mathrm{NC}$ & \\
\hline Fiber volume & Fb.V/TV (\%) & 0.00 & 0 \\
\hline
\end{tabular}

$N C$ not calculated

${ }^{a}$ Reference data from iliac crests of female over 71 years old

the patient can now walk independently without crutch.

All procedures followed were in accordance with the ethical standards of the responsible committee on human experimentation (institutional and national) and with the Helsinki Declaration of 1975 , as revised in 2000 and 2008. Informed consent was obtained from the patient for being included in the case report.

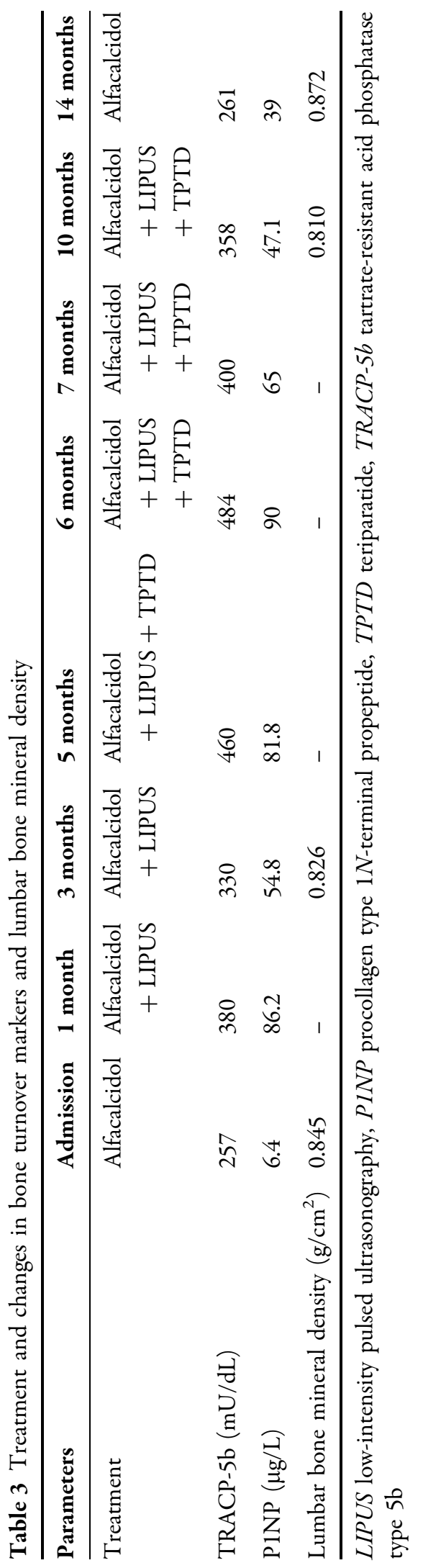



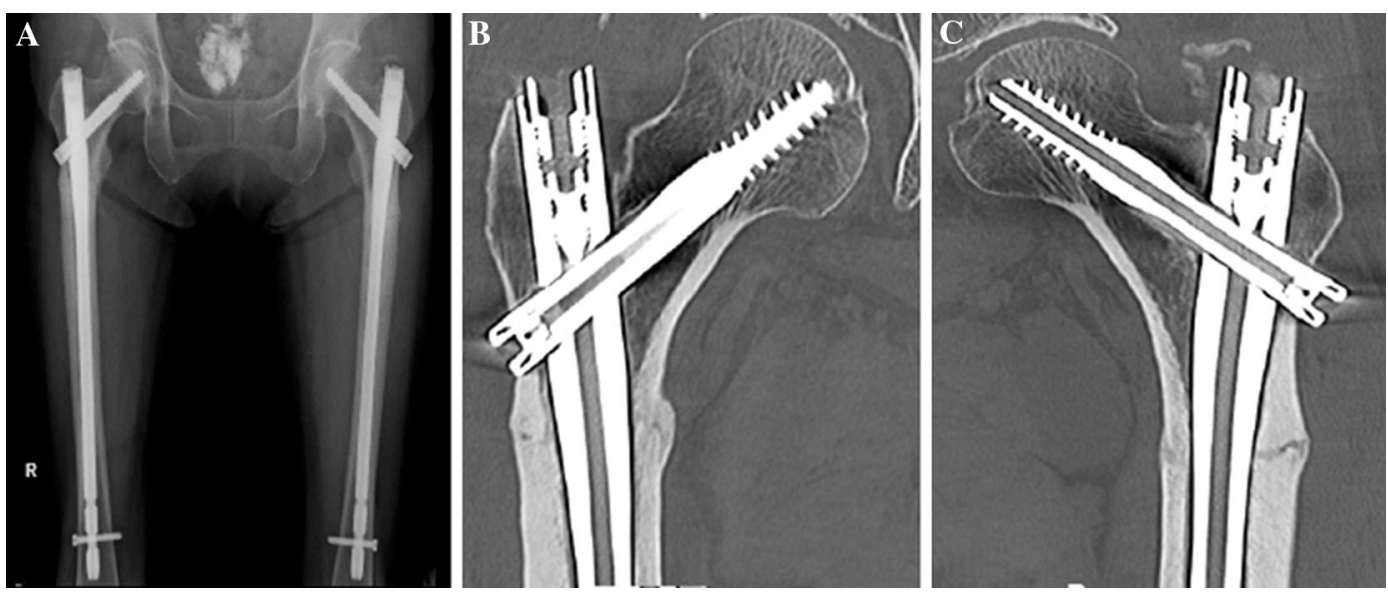

Fig. 5 Radiograph and CT imaging after 3 months of TPTD therapy. a Radiograph imaging after 3 months of TPTD therapy shows complete union of fractures. $\mathbf{b}$ CT imaging after 3 months of TPTD therapy shows complete

\section{DISCUSSION}

The occurrence of AFFs associated with severely suppressed bone turnover (SSBT) in osteoporotic patients receiving bisphosphonates over prolonged periods was first reported in 2005 [7]. A task force from the American Society for Bone and Mineral Research (ASBMR) defined the major and minor features of AFFs [8], recommending that all major features should be present to confirm a diagnosis of AFF. Bisphosphonates effectively reduce the risk of femoral neck fractures in elderly women, but when continued for more than 5 years, the therapy can result in excessive suppression of bone turnover, thus increasing the risk of AFFs [9].

The present case had an approximately 6-year history of alendronate therapy and subtrochanteric fractures with the major features designated by ASBMR; therefore, the case was diagnosed as having bisphosphonateassociated AFFs. Although urine $\mathrm{N}$-telopeptide of type I collagen and serum TRACP-5b levels were normal, the patient's serum P1NP was low, union of the right thigh fracture. c CT imaging after 3 months of TPTD therapy shows complete union of the left thigh fracture. CT computerized tomography, TPTD teriparatide

suggesting reduced bone formation. Bone biopsy was performed at the intramedullary nail insertion site and undecalcified thin sections were made for trabecular bone histomorphometry. The data revealed a decrease in bone formation parameters (wall thickness, osteoid volume, osteoid surface, osteoid thickness, osteoblast surface) as well as a decrease in bone resorption parameters (eroded surface, osteoclast surface). It was speculated that the patient's bone turnover was extremely low in the proximal femur of this patient, and that SSBT attributed to alendronate use for more than 5 years led to their AFFs and delayed union. On the other hand, Zanchetta et al. [10] reported that there were no distinctive microarchitecture features in the peripheral skeleton of a female patient who had suffered AFF while receiving bisphosphonate treatment. So, other factors including patient characteristics and instability of osteosynthesis might be concerned with AFFs and delayed union.

Union of both femur fractures was confirmed after 3 months of TPTD treatment. It was 
thought that TPTD stimulated the suppressed bone formation caused by long-term use of bisphosphonates. Two types of TPTD injection are available for treatment of osteoporosis in patients with a high risk of fracture in Japan: once-daily $20 \mu \mathrm{g}$ or once-weekly $56.5 \mu \mathrm{g}$. It was thought that the increased bone formation owing to TPTD may have accelerated fracture healing and improved bone union. A search of the literature revealed several other similar clinical cases that were successfully treated with TPTD [1-6]. Aspenberg et al. [11] showed a shorter healing time in postmenopausal women with dorsally angulated distal radial fractures who were treated once daily with TPTD $20 \mu \mathrm{g}$, compared with women treated with placebo. However, no difference in fracture healing time was observed between women treated once daily with TPTD $40 \mu \mathrm{g}$ and women treated with placebo [11]. Therefore, it appears that the dose of TPTD is important to achieve accelerated fracture repair. Although the patient in the current study received onceweekly TPTD, the overall dose received is considered closer to the once-daily $20 \mu \mathrm{g}$ dose than to the once-daily $40 \mu \mathrm{g}$ dose. Chiang et al. [12] showed that once-daily TPTD $20 \mu \mathrm{g}$ improved the bone quality and healing of AFFs associated with bisphosphonate therapy, while Mitani [13] described a case in Japan showing nonunion of a femoral neck fracture who, like the patient in the current study, benefited from once-weekly treatment with TPTD $56.5 \mu \mathrm{g}$. Collectively, these findings [13], together with the findings in the current case, suggest that fracture repair might be accelerated by once-weekly treatment with TPTD $56.5 \mu \mathrm{g}$, or once-daily treatment with TPTD $20 \mu \mathrm{g}$. Warden et al. [14] reported in animal model that TPTD and LIPUS have contrasting additive effects during fracture healing, and the patient in the current case treated with TPTD and LIPUS, so the fracture repair might be due to the combination therapy. Based on the authors' experience, it is suggested that once-weekly TPTD treatment is beneficial for improving the healing of AFFs showing delayed union as well as other fracture treatment methods including electrical stimulation, bone graft, bone morphogenetic proteins, and LIPUS.

\section{CONCLUSION}

Suppressed bone formation caused by long-term bisphosphonate therapy can delay the union of fractures in osteoporotic patients. Although TPTD administration has been shown to accelerate the healing in such patients, it is not clear if daily or weekly treatments are required. The authors demonstrate in this report that weekly treatments are sufficient and should be considered for treating patients with AFFs with delayed union.

\section{ACKNOWLEDGMENTS}

All named authors meet the ICMJE criteria for authorship for this manuscript, take responsibility for the integrity of the work as a whole, and have given final approval to the version to be published. Article processing charges for this study were funded by Asahikasei Pharma Corporation.

Conflict of interest. F. Fukuda, N. Kurinomaru, and A. Hijioka, declare no conflict of interest.

Compliance with ethical guidelines. All procedures followed were in accordance with the ethical standards of the responsible committee on human experimentation (institutional and national) and with the 
Helsinki Declaration of 1975, as revised in 2000 and 2008. Informed consent was obtained from the patient for being included in the case report.

Open Access. This article is distributed under the terms of the Creative Commons Attribution Noncommercial License which permits any noncommercial use, distribution, and reproduction in any medium, provided the original author(s) and the source are credited.

\section{REFERENCES}

1. Gomberg SJ, Wustrack RL, Napoli N, Arnaud CD, Black DM. Teriparatide, vitamin D, and calcium healed bilateral subtrochanteric stress fractures in a postmenopausal woman with a 13-year history of continuous alendronate therapy. J Clin Endocrinol Metab. 2011;96:1627-32.

2. Carvalho NN, Voss LA, Almeida MO, Salgado CL, Bandeira F. Atypical femoral fractures during prolonged use of bisphosphonates: short-term responses to strontium ranelate and teriparatide. J Clin Endocrinol Metab. 2011;96:2675-80.

3. Huang HT, Kang L, Huang PJ, et al. Successful teriparatide treatment of atypical fracture after long-term use of alendronate without surgical procedure in a postmenopausalwoman: a case report. Menopause. 2012;19:1360-3.

4. Mastaglia S, Aguilar G, Rossi E. Rapid resolution with teriparatide in delayed healing of atypical fracture associated to long-term bisphosphonate use. J Bone Miner Res. 2012;27(Suppl 1):S1.

5. Cheung AM, Bleakney R, Kahn A, et al. Effect of teriparatide on fracture healing in patients with non-displaced incomplete atypical femur fractures. J Bone Miner Res. 2012;27(Suppl 1):S1.

6. Miller PD, McCarthy E. Quantitative bone histomorphometry in patients with bisphosphonate-associated atypical subtrochanteric femur fractures before and after 12 months of teriparatide. J Bone Miner Res. 2012;27(Suppl 1):S1.

7. Odvina CV, Zerwekh JE, Rao DS, Maalouf $\mathrm{N}$, Gottschalk FA, Pak CY. Severely suppressed bone turnover: a potential complication of alendronate therapy. J Clin Endocrinol Metab. 2005;90:1294-301.

8. Shane E, Burr D, Ebeling PR, et al. Atypical subtrochanteric and diaphyseal femoral fractures: second report of a Task Force of the American Society for Bone and Mineral Research. J Bone Miner Res. 2014;29:1-24.

9. Park-Wyllie LY, Mamdani MM, Juurlink DN, et al. Bisphosphonate use and the risk of subtrochanteric of femoral shaft fractures in older women. JAMA. 2001;305:783-9.

10. Zanchetta MB, Diehl M, Buttazzoni $M$, et al. Assessment of bone microarchitecture in postmenopausal women on long term bisphosphonate therapy with atypical fractures of the femur. J Bone Miner Res. 2013. doi:10.1002/ jbmr.2107.

11. Aspenberg P, Genant HK, Johansson $\mathrm{T}$, et al. Teriparatide for acceleration of fracture repair in humans: a prospective, double-blind study of 102 postmenopausal women with distal radial fractures. J Bone Miner Res. 2010;25:404-14.

12. Chiang CY, Zebaze RM, Ghasem-Zadeh A, IulianoBurns S, Hardidge A, Seeman E. Teriparatide improves bone quality and healing of atypical femoral fractures associated with bisphosphonate therapy. Bone. 2013;52:360-5.

13. Mitani Y. Effective treatment of a steroid-induced femoral neck nonunion with a once-weekly administration of teriparatide in a rheumatoid patient: a case report. Arch Osteoporos. 2013;8:131.

14. Warden SJ, Komatsu DE, Rydberg J, Bond JL, Hassett SM. Recombinant human parathyroid hormone (PTH 1-34) and low-intensity pulsed ultrasound have contrasting additive effects during fracture healing. Bone. 2009;44:485-94. 